\title{
Neue Forschungsförderung der AOTrauma Deutschland: von der Projekt- zur Nachwuchsförderung
}

\author{
Georg Duda, Philip Wilbrandt
}

Die AOTrauma Deutschland stellt ihre Forschungsförderung zum Jahr 2016 um. Anstelle der Förderung konkreter Forschungsprojekte wird der Fokus eindeutig auf die Nachwuchsförderung gelegt.

Die deutsche AO hat bisher die Forschung im Rahmen von 2 Calls mit Mitteln in Höhe von ca. $75000 €$ pro Jahr gefördert. Die eingereichten Anträge wurden begutachtet und mit bis zu $15000 €$ pro Projekt gefördert. Von jungen Wissenschaftlern und Klinikern eingereichte Arbeiten wurden bevorzugt.

Ab diesem Jahr ist das primäre Ziel die Förderung des klinischen Nachwuchses in klinischer und experimenteller Forschung durch Mentoring und eine Anschubfinanzierung. Junge (bis zum Alter von 35 Jahren), klinisch aktive Unfallchirurgen, die in Deutschland an Universitätskliniken, regionalen und kommunalen Krankenhäusern tätig sind, können sowohl klinische als auch experimentelle Forschungskonzepte aus den Bereichen Traumatologie, MKG und VET einreichen.

Zweimal im Jahr - 1. März und 1. September - werden von der AOTD-Forschungskommission Arbeiten ausgewählt und die antragstellende Person eingeladen, ihre Arbeit auf einer wissenschaftlichen Sitzung (entweder AO-Jahrestagung im Mai oder DKOU im Oktober) vorzustellen und $\mathrm{zu}$ verteidigen. Am Ende der Sitzung werden zwei Drittel der eingeladenen Personen mit einer Anschubfinanzierung in Höhe von bis zu $12000 €$ für ihre Arbeit prämiert.

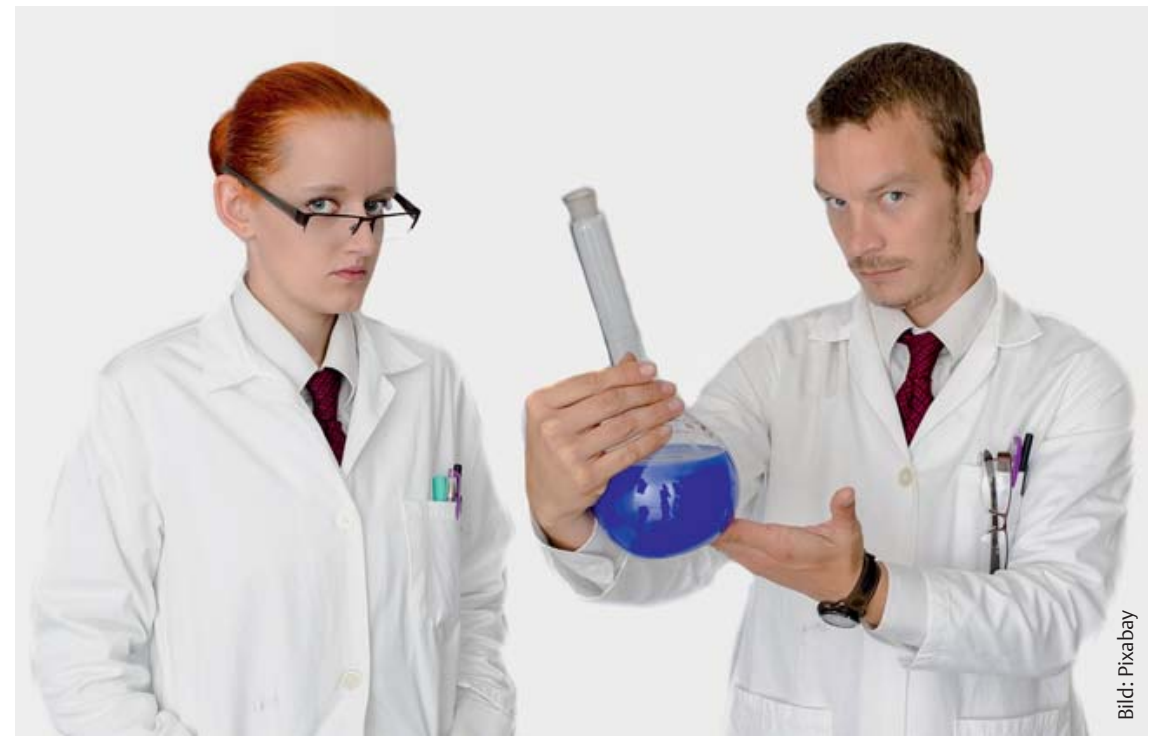

Aus den Bewerbungen werden Kandidaten zur Auswahlsitzung AO-Forschungsförderung eingeladen (Anwesenheit ist Pflicht). Jeder Kandidat hat 6 Minuten, um die Arbeit vorzustellen. Danach folgt eine Diskussion und die Auswahl.

\section{Bewerberkriterien}

- Kliniker

- Nachwuchs (bis 35 Jahre)

- Unterstützung vom Chef

- AO-Mitglied

Alle weiteren Informationen inkl. dem Antragsformular finden Sie auf der Homepage der Deutschen AO.

Der Wissenschaftspreis der Deutschen AO bleibt unverändert und wird wie bisher einmal pro Jahr ausgeschrieben.
Die Bewerbungsfrist läuft bis zum 31.08.2016.

Prof. Dr.-Ing. Georg Duda

Research Officer

Julius Wolff Institute and Center for Musculoskeletal Surgery Berlin-Brandenburg Center for Regenerative Therapies Charité - Universitätsmedizin Berlin Augustenburger Platz 1 13353 Berlin

\section{Philip Wilbrandt}

AOTrauma Deutschland

Langenbeck-Virchow-Haus

Luisenstraße 58/59

10117 Berlin 